\title{
DE LOS ELEMENTOS PERFORADOS AL COBOGÓ: HISTÓRICO DE USO EN LA ARQUITECTURA BRASILERA Y CONSIDERACIONES SOBRE SU ADAPTACIÓN AL CLIMA
}

\author{
Brick vents to Cobogó: history of use in Brazilian architecture and considerations \\ regarding its adaptation to climate
}

\author{
Darwin Onésimo Jaime Camacho ${ }^{1}$ \\ Universidad Federal de Integración Latinoamericana, Foz de Iguazú, PR, Brasil, darwin.camacho@aluno.unila.edu.br \\ Helenice María Sacht ${ }^{2}$ \\ Universidad Federal de Integración Latinoamericana, Foz de Iguazú, PR, Brasil, helenice.sacht@unila.edu.br
}

Egon Vettorazzi 3

Universidad Federal de Integración Latinoamericana, Foz de Iguazú, PR, Brasil, egon.vettorazzi@unila.edu.br

\section{Resumen}

En las últimas décadas, debido a las alteraciones climáticas y la posible escasez de energía, muchos son los investigadores en los campos de la arquitectura e ingeniería civil que encuentran alternativas y estrategias pasivas que puedan mitigar esos efectos y establecer una relación entre arquitectura y clima. En este contexto, los elementos perforados, específicamente el cobogó, en el caso de gran parte de Brasil son una alternativa arquitectónica que puede ser usada para minimizar las condiciones climáticas adversas, pues pueden proporcionar ventilación natural permanente, así como protección solar, e iluminación natural. Delante de estos aspectos, se presenta una revisión bibliográfica sobre el origen, evolución e importancia de los elementos perforados (cobogós) y algunos aspectos que lo relacionan como elemento arquitectónico que auxilia en la adaptación al clima, influenciando en las condiciones de confort ambiental y eficiencia energética. Se utilizó el método de investigación bibliográfica - documental, con base en la búsqueda de fuente de datos académicos. Fueron analizados documentos en español, portugués e inglés, publicados de 2014 a 2016. Posteriormente fue aplicado el Teorema de Gauss e por fin, realizado el análisis de títulos y resúmenes de los documentos más relevantes sobre el tema. Se observó la importancia de los elementos vazados para la arquitectura, y la necesidad de la realización de nuevos estudios para evaluación de desempeño de este dispositivo, además de cuestiones estéticas muy abordadas.

Palabras- clave: Cobogós. Elementos perforados. Ventilación natural. Eficiencia energética.

\begin{abstract}
In the last decades, due to climate change, and to energy shortages, researchers in the architecture and civil engineering areas try to find alternatives and passive strategies to mitigate these effects and establish a relationship between architecture and the climate. In this context, hollow elements (brick vents), called Cobogó in Brazil are an architectural element that can be used to minimize adverse climatic conditions, and it can provide permanent natural ventilation, sun protection, and daylight. Based on this aspect, a bibliographic review is presented about the origin, evolution, and importance of the hollow elements (brick vents), (cobogós) and some aspects that relate it as an architectural element that assists in adaptation to climate, influencing on the conditions of environmental comfort and energy efficiency. A bibliographic-documentary research method was used, using a search engine in academic databases. Documents in Spanish, Portuguese and English, published from 2014 to 2016, were analyzed. Posteriorly, Gauss Theorem was applied and, finally, the titles and abstracts of the most relevant documents on the subject were examined. The importance of the brick vents on architecture and the need to carry out new research to evaluate the performance of the device, beyond the aesthetic approaches, were observed..
\end{abstract}

Keywords: Cobogós. Brick vents. Natural ventilation. Energy efficiency.

How to cite this article:

CAMACHO, Darwin Onésimo Jaime; SACHT, Helenice Maria; VETTORAZZI, Egon. De los elementos perforados al cobogó: histórico de uso en la arquitectura brasilera y consideraciones sobre su adaptación al clima. PARC Pesquisa em Arquitetura e Construção, Campinas, SP, v. 8, n. 3, p. 205-216, set. 2017. ISSN 1980-6809. Disponível em: <https://periodicos.sbu.unicamp.br/ojs/index.php/parc/article/view/8650237>. Acesso em: 27 jan. 2018. doi:http://dx.doi.org/10.20396/parc.v8i3.8650237. 


\section{Introducción}

En América Latina estudios realizados por la organización ELLA Network (2013) demuestran que los edificios consumen el $42 \%$ de energía eléctrica, producen el $25 \%$ de las emisiones del $\mathrm{CO} 2$ y el $65 \%$ de los residuos sólidos. En la misma línea de pensamiento Brasil es el sexto país a nivel mundial que consume energía, quedando atrás de China, Estados Unidos, India, Rusia y Japón. Ya en América Latina Brasil ocupa el primer lugar en consumo de energía (IEA, 2018).

De esta forma, al analizar datos más recientes, disponibles sobre el consumo energético de Brasil (EPE, 2017), se observa un porcentaje significativo en el sector residencial, lo que justifica buscar iniciativas que permitan estudiar estrategias para la reducción del consumo y mejorar la eficiencia energética.

En el año 2016, como se observa en la figura 1, el consumo de energía eléctrica fue de $25,60 \%$ para el sector residencial, $17,20 \%$ para el sector comercial y $8,30 \%$ para el sector público. Por lo tanto, se observa que el consumo de energía eléctrica en las edificaciones corresponde a un $51,10 \%$ del consumo total, sumando los tres sectores antes mencionados (BRASIL, 2017).

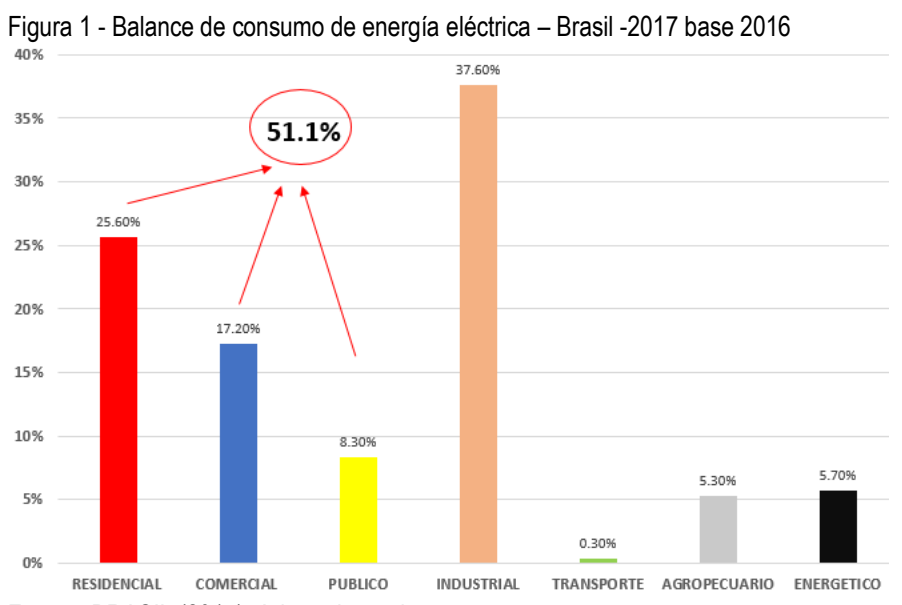

Fuente: BRASIL (2017). Adaptado por los autores

Sin embargo, en las últimas décadas, la intensificación de los problemas ambientales provenientes del calentamiento global, han traído consigo, tanto en la arquitectura como en la ingeniería, la toma de conciencia y la preocupación, por establecer una adecuada integración, entre el ambiente construido y el medio ambiente; con el objetivo, de proponer estrategias, que generen un mejor desempeño energético en las edificaciones y un mayor confort térmico. Además, se observa una tendencia en el rescate de soluciones y estrategias bioclimáticas y/o vernáculas que desde el inicio de la humanidad han sido aplicadas en forma intuitiva, contribuyendo de esta forma para un mejor confort ambiental, así como la adaptación de la arquitectura al clima.
Dicha relación entre arquitectura y clima, como lo manifiesta Araújo (2011), en la actualidad debe adoptar técnicas constructivas, que generen soluciones viables y económicas, pudiendo ser utilizadas por toda la población, con el objetivo de lidiar con las condiciones ambientales que cada región y/o ciudad presenta, contribuyendo para la realización de una arquitectura de menor impacto ambiental.

Desde el inicio de la humanidad, el ser humano ha aprendido a construir su hábitat, y para eso tuvo que dar forma a ciertos materiales y aprender a usarlos, para que éstos puedan atender sus necesidades, cumpliendo funciones como seguridad, comodidad, buena apariencia y un óptimo aprovechamiento de la naturaleza, en cuestiones de confort y eficiencia energética.

Según Guerra (2013), la eficiencia energética aplicando estrategias pasivas de iluminación y ventilación, es uno de los aspectos necesarios a tomar en cuenta para una optimización de las estrategias sustentables; este aspecto debe estar relacionado con la económica energética y la utilización de energías renovables.

Delante de estos aspectos, se tiene como estrategia bioclimática, el uso de elementos perforados en la arquitectura, tanto para el aprovechamiento de la iluminación, control de la radiación solar directa, así como de la ventilación natural. Dentro de este conjunto de elementos perforados se encuentra el cobogó, un elemento arquitectónico propio de la arquitectura brasilera, pero que ha sido utilizado mundialmente, especialmente en la arquitectura latinoamericana, tomando diferentes formatos, materiales y nombres como combogó, ladrillos perforados, calados, celosías, etc.

\section{Elementos Vazados}

Los elementos perforados, son componentes constructivos de cerramiento y protección que están categorizados dentro de un conjunto de elementos arquitectónicos (Figura 2); y como lo manifiesta Bittencourt (1995) y Ching (2000), tienen por finalidad, ventilar y filtrar la iluminación natural dentro de un ambiente, así como la protección solar.

Bittencourt (1995), apunta aún que estos elementos tienen sus orígenes en la arquitectura vernácula y/o tradicional, cuyas raíces se encuentran en el medio oriente ya que las inclemencias del clima, los condujo a la utilización de materiales y técnicas que proporcionen un mejor aprovechamiento de los recursos naturales.

Basados en los aspectos anteriormente mencionados, el presente artículo presenta un breve estado del arte del uso de los elementos perforados hasta el cobogó en la Arquitectura Brasilera, así como las tendencias y 
perspectivas de su uso actual, para el confort ambiental y eficiencia energética.

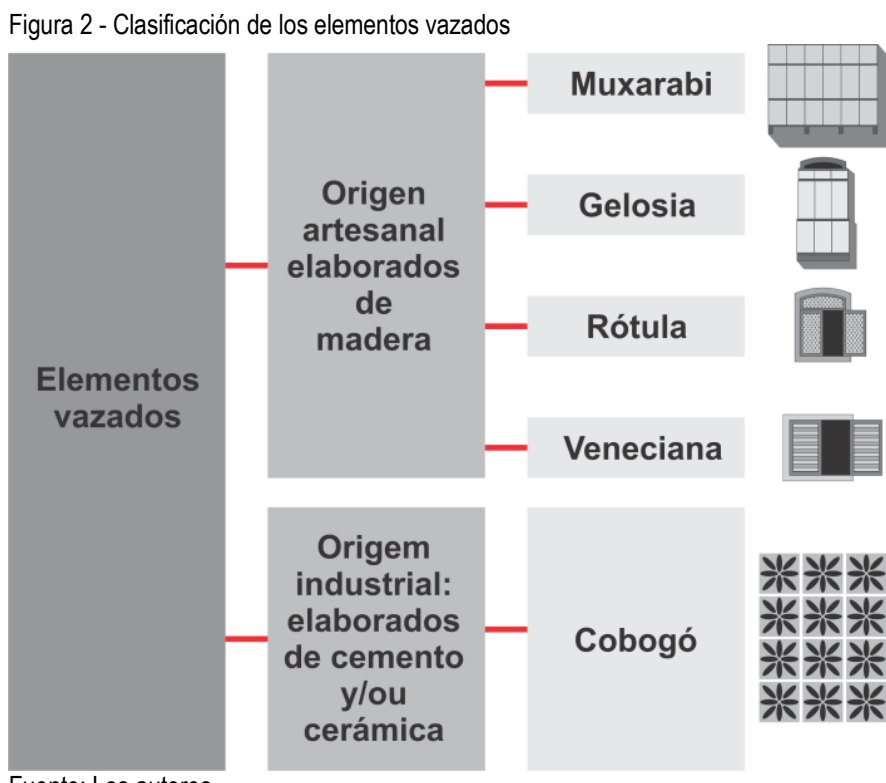

Fuente: Los autores

\section{Metodología}

La metodología del proyecto es considerada por Gomez (2004), como la organización de conocimientos, procedimientos $\mathrm{y}$ herramientas utilizadas para el desenvolvimiento de productos, en que la eficiencia y eficacia de éstos, necesita saber; "qué", "cuándo" y "cómo hacer". De la misma manera Marconi y Lakatos (2003), manifiestan que la metodología es un conjunto de actividades sistemáticas y racionales, que permiten alcanzar el objetivo planteado en un estudio de investigación.

La metodología por aplicar en este artículo es de tipo bibliográfica - documental, que según Marconi e Lakatos (2003), este tipo de metodología tiene como finalidad investigar acontecimientos y/o procesos del pasado para luego verificar y analizar su influencia en la sociedad de hoy.

En este contexto, la búsqueda de la información para este artículo fue determinada por la utilización de fuentes de datos tales como Google Académico, quien dará soporte científico a esta pesquisa. Para tal efecto se establecieron siete palabras claves (Figura 3) referidas al tema a investigar; dichos términos están relacionados con la arquitectura e ingeniería. De esta búsqueda resultaron 3498 documentos; como ese número era significativo, se determinaron criterios de selectividad para reducir el número de documentos a ser analizados. Para tal efecto se aplicaron cinco filtros.
- Filtro 1 (Idioma): Los documentos a investigar solo estarían en español, portugués e inglés. De este filtro resultaron 3475 documentos.

- $\quad$ Filtro 2 (Año de Publicación): Por la importancia del tema se determinó que los documentos a analizar deberían ser actuales, para ello se limitó la búsqueda entre los años 2014 a 2016. De este filtro resultaron 971 documentos.

- $\quad$ Filtro 3 (Aplicación del Teorema de Gauss): Dentro de las metodologías de investigación, está la aplicación de métodos estadísticos para determinar una muestra significativa. Como se observa después de aplicados los filtros anteriores el número de documentos era aún significativo, por tal motivo se estableció la aplicación del teorema de Gauss, con un nivel de confiabilidad del $95 \%$ y un margen de error de 5\%. De este filtro resultaron 644 documentos llamadas muestras.

- Filtro 4 (Lectura de Títulos): De los documentos seleccionados en el tercer filtro se inició la lectura de los títulos y aquellos que se encuadraron dentro del objetivo de esta pesquisa fueron seleccionados, resultando 160 documentos.

- Filtro 5 (Lectura de Resúmenes): En este filtro se organizó la documentación por categorías (tecnología e historia), para luego leer cada uno de los resúmenes e ir filtrando la información válida para esta pesquisa, en este filtro resultaron 78 documentos aptos para realizar el análisis completo de cada uno de ellos.

Cabe resaltar que los documentos que sobraron después de aplicados los filtros no fueron seleccionados porque no trataban aspectos históricos, informaciones técnicas de uso y materiales sobre el tema a desenvolver en esta investigación.

Con los 78 documentos organizados y filtrados por su nivel de relevancia e interés (Figura 3), se prosiguió a leer cada uno de los artículos seleccionados y de esta manera se elaboró el estado del arte sobre el tema elementos perforados (cobogós).

Como se visualiza en la figura 3, fueron un total de 3498 documentos, encontrados en la base de datos Google académico; de los cuales la palabra clave "elementos perforados" fue la que más apareció en los documentos, ocupando así un $28,6 \%$, ya la palabra "ladrillo perforado" ocupo un $19,2 \%$. Sin embargo, la palabra que menos apareció en la búsqueda fue "brick vents" con un $1,1 \%$, seguido de la palabra "natural ventilation devices" con un $3,5 \%$. En consecuencia, estos datos nos revelan que este tema obtiene mucho más relevancia en idiomas tanto en portugués como en español. 


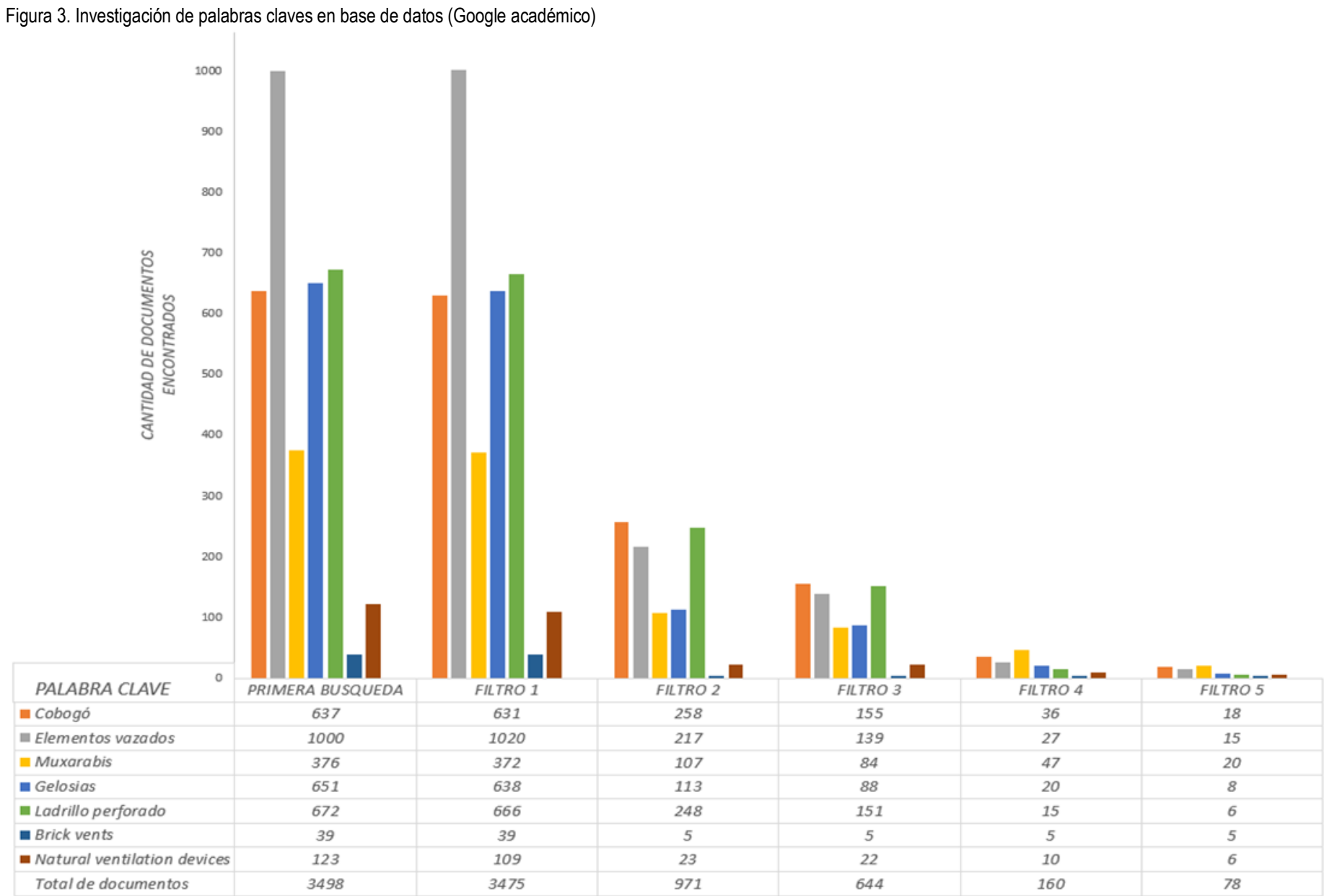

Fuente: Los autores.

\section{Origen y Evolución de los Elementos Perforados en la Arquitectura Brasilera}

Si se remonta a épocas pasadas, el ser humano a lo largo del tiempo, independientemente del territorio en el que habitaba, desarrolló estrategias pasivas para obtener conforto ambiental, siendo éstas, propias de cada cultura, y estaban ligadas al entendimiento e integración entre arquitectura y clima. Este tipo de estrategias, soluciones y técnicas constructivas, se perfeccionaron más, gracias al contacto intercultural que produjo la expansión territorial de cada cultura, convirtiéndolas en adecuadas soluciones arquitectónicas, que promovían el desenvolvimiento de estrategias pasivas.

A lo largo de la historia, es común dentro de la arquitectura, visualizar el empleo de elementos perforados, para el aprovechamiento de iluminación, ventilación natural, control solar y además de brindar privacidad en el ambiente interno.

Esta utilización puede ser ejemplificada en las tradiciones orientales, musulmanas e islámicas, así como también en la arquitectura latinoamericana, teniendo un especial énfasis en la arquitectura colonial y arquitectura moderna de Brasil (PAULERT, 2012).

En la arquitectura realizada por el mundo islámico, dos fueron los principales problemas a resolver por constructores y arquitectos; el primero, conseguir protección contra el inclemente sol, y el segundo, refrescar el ambiente interno. Dichas preocupaciones fueron resueltas gracias a un buen planeamiento realizado con respecto a layout, localización de los espacios en las edificaciones y sobre todo el uso de elementos arquitectónicos que lograron amortiguar los efectos climáticos (EL-SHORBAGY, 2010).

Dentro del conjunto de los elementos arquitectónicos, utilizados en la arquitectura islámica y musulmana, encontramos a los elementos perforados, tales como el muxarabi (Figura 4), un elemento compuesto por viguetas o pantallas de madera siguiendo patrones geométricos; estos, estaban colocados en los balcones de las edificaciones con el objetivo de controlar el paso de la luz y el flujo de aire natural, reduciendo la temperatura interior así como asegurar la privacidad de las mujeres; aspecto de vital importancia en la cultura islámica (FICARELLI, 2009; EL-SHORBAGY, 2010; FATHY, 2010). 


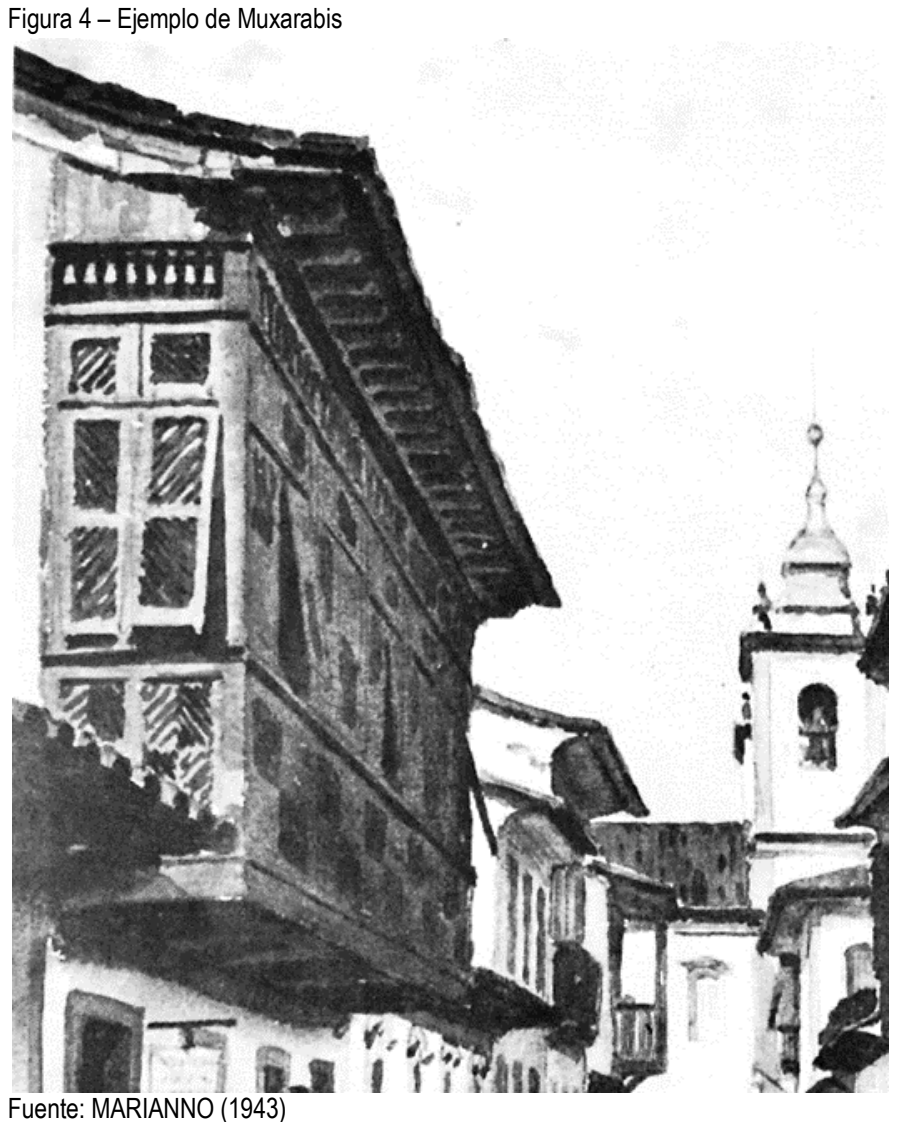

Otros elementos arquitectónicos islámicos, son las rótulas y celosías; al igual que los muxarabis pueden presentar definiciones diferentes por investigadores y arquitectos; sin embargo Rabbat (1988), Marianno (1943), Colin (2010), concuerdan que las rótulas (Figura 5) son cerramientos compuestos por dos hojas de abrir, hechos de viguetas de ripias de madera, colocados rasantemente en el lado externo de las ventanas; ya las celosías (figura 6) son como una especie de caja sobrepuesta hechas de viguetas de madera, colocadas preferiblemente en las ventanas de los pisos superiores.

Ya en América del Sur; Brasil, como consecuencia de la colonización iniciada en 1530, su arquitectura colonial como lo manifiesta Mello (1974), Gomes (2004), estuvo influenciada por la arquitectura portuguesa y esta a su vez por la arquitectura islámica, ya que Portugal estuvo dominada por la civilización musulmana.

Los elementos perforados como muxarabis, celosías y rótulas, herencias musulmanas, fueron rápidamente introducidos en las edificaciones populares de Brasil; y como lo manifiesta Marianno (1943) y Pinto y Freyre (1958), estos se adaptaban al medio ambiente local, siendo utilizados en casas sin patios y jardines, pues a través de estos trenzados de madera, la ventilación de las calles penetraba en el interior del ambiente, promoviendo confort térmico y lumínico.
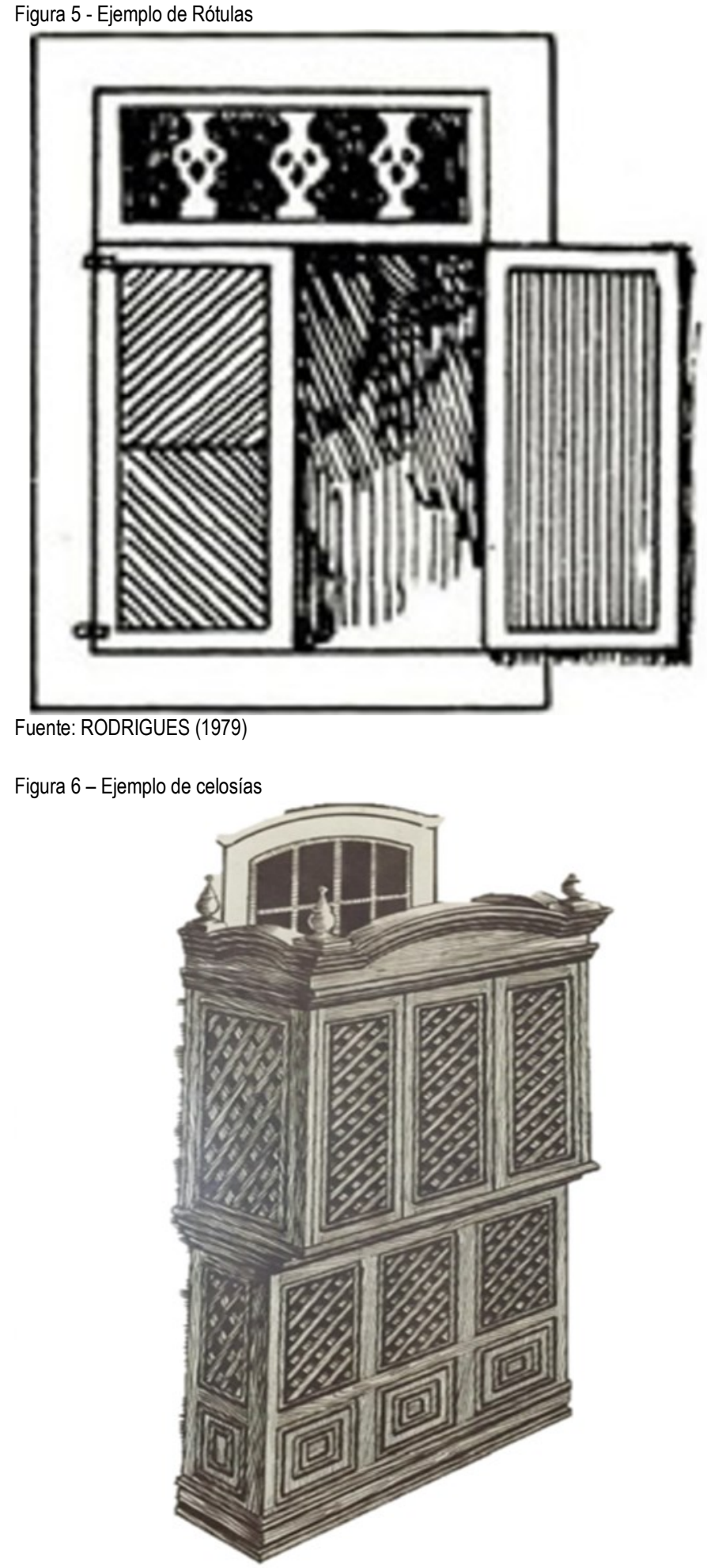

Fuente: MARIANNO (1943)

Un testimonio vivo de la transposición cultural, adaptada al territorio y clima brasileño, con influencia islámica, se encuentra en Diamantina - MG especialmente en la Casa Chica da Silva (Figura 7) y la Casa de Rótulas en Pilar de Goiás - GO (figura 8). Allí se aprecia la utilización de viguetas de madera en rotulas, celosías y muxarabis protegiendo las aberturas del sol directo. 


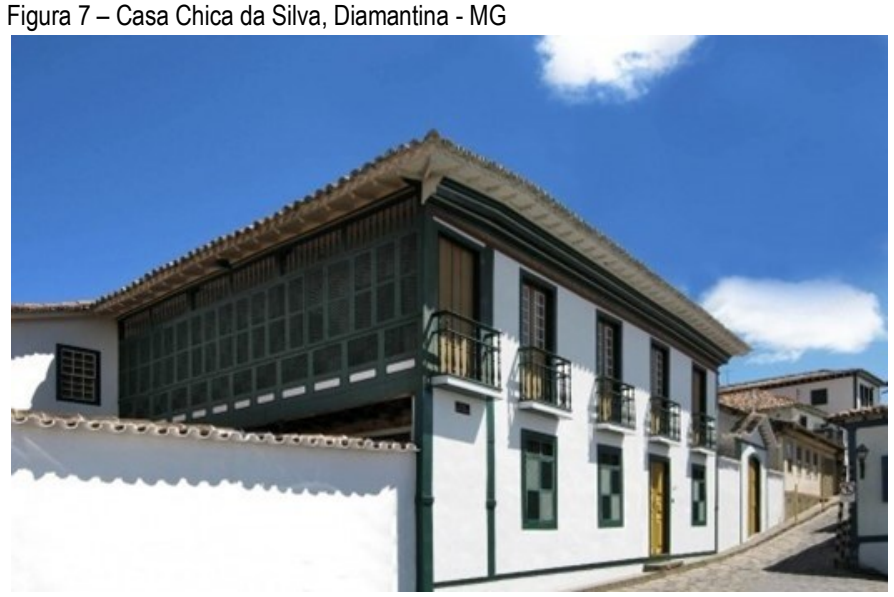

Fuente: LEMOS (2012)

Figura 8 - Casa de Rótulas, Pilar de Goiás - GO

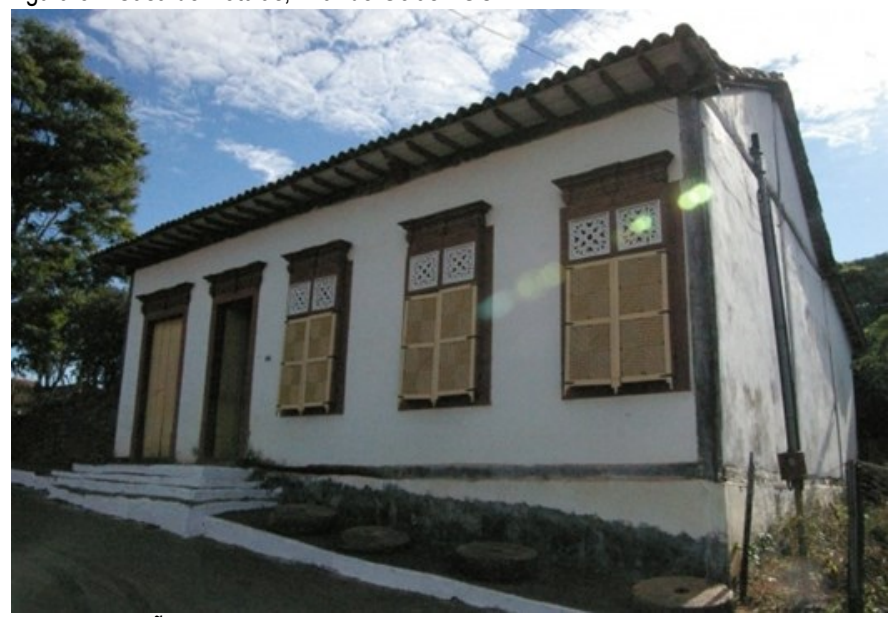

Fuente: GALVÃO (2008)

Sin embargo, en 1809, la arquitectura colonial brasilera, vivió la primera y más grande crisis; oficialmente, como lo manifiesta Marianno (1943), es decretado por la familia real portuguesa, la muerte de los más preciosos elementos arquitectónicos con influencia islámica; en vez de muxarabis, rotulas y celosías, se colocaron barandas de hierro batido, o de madera, con balaustres torneados, y en las aberturas se colocaron ventanas con vidrio llamadas guillotina.

Con el cumplimiento obligatorio de ese decreto, comienza a ser implantada una arquitectura influenciada por patrones europeos. Esta influencia, es visualizada con más énfasis en las primeras décadas del siglo XX; aquí los arquitectos, constructores e ingenieros comienzan a realizar simples reproducciones de la arquitectura europea sin preocupación con la cultura local y el clima. En consecuencia, nacen arquitecturas historicistas, una valorización y reproducción de la arquitectura oficial europea que hicieron prevalecer elementos industrializados con una principal función estética, abandonando la relación entre arquitectura y clima (MINDLIN, 1956).
Paralelamente a estos acontecimientos nace la arquitectura moderna brasilera, pero a diferencia de las arquitecturas historicistas, como lo manifiesta Goodwin (1943), ésta, logró adaptar los conceptos modernos universales a la realidad climática local, proponiendo innovadoras soluciones arquitectónicas que incluían un lenguaje proyectual con carácter propio.

Mindlin (1956), define a la producción moderna brasilera del siglo XX, como la arquitectura del sol; una arquitectura que trabaja con propuestas basadas en estudios de control de luz, de insolación y sobre todo en la conexión entre modernidad y regionalismo.

En la misma línea de pensamiento Nery (1999), manifiesta que en 1926 Gilberto Freyre presenta el Manifiesto Regionalista en el Primer Congreso Brasilero de Regionalismo; este acontecimiento marco la reflexión sobre la necesidad de conciliar la tradición con la modernidad. De esta forma se visualiza la necesidad de no solo copiar modelos extranjeros, y sí; proponer soluciones donde se pueda producir arquitectura moderna, rescatando lo local y/o regional.

Localizándonos geográficamente, en la región Nordeste de Brasil, específicamente en Recife, en el siglo XX, debido a la industrialización y factores políticos, económicos y socioculturales, hubo una llegada de arquitectos inmigrantes provenientes de Europa, estos; hicieron parte de la producción arquitectónica moderna, pero tomando como pauta fundamental, la realidad local tropical adaptándose de esta manera, a las características físicas y sociales del medio (AFONSO, 2001; SILVA, 1988).

En ese contexto y motivados por la búsqueda de una identidad arquitectónica nacional, nace 1929 el componente arquitectónico llamado cobogó. ¿Pero, "que es este elemento" y, "cuáles son sus orígenes"?

\section{Cobogós}

El cobogó tiene origen en la arquitectura tradicional (Figura 9) y nace de la necesidad de adaptar la arquitectura al clima con un lenguaje compositivo que represente la modernidad de los nuevos tiempos (Figura 10) que trae como resultado una solución constructiva adecuada, a partir de la combinación entre modernidad (concreto) y técnicas tradicionales (muxarabis, celosías y rótulas).

Su origen se remonta en el año 1929, año en el que fue patentado, en la ciudad de Recife - Pernambuco y su nombre, deriva de sus tres creadores, los ingenieros: Amadeu Oliveira Coimbra, Ernest agosto Boeckmann y Antonio de Gois (FERREIRA, 2004; BRAGA, 2010).

Pallone (2010), considera que las reinterpretaciones modernas bien sucedidas, son aquellas que nos conmueven, no solo por su nivel estético, si no por su gran compromiso con el pasado y con lo regional; en ese 
sentido y apoyado de investigadores como Afonso (2001), se puede afirmar que el cobogó es una reinterpretación generada a partir de la evolución de soluciones regionales basadas en los muxarabis, celosías y rotulas, empleadas en la arquitectura vernácula - colonial brasilera y que estos a su vez tuvieron influencia musulmana.

Figura 9 - Origen del cobogó: influencia de elementos tradicionales

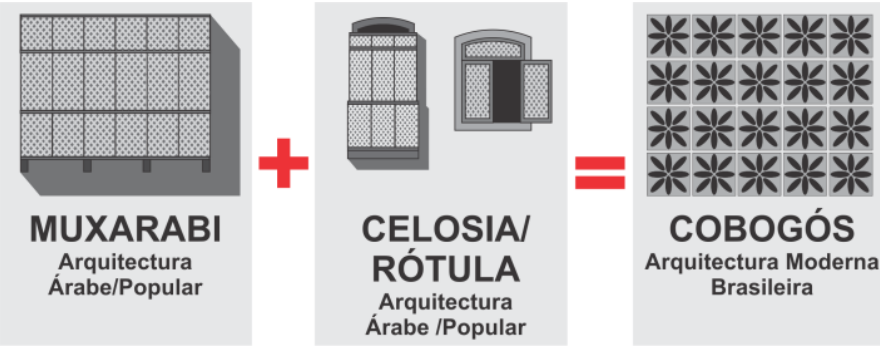

Fuente: Los autores

Figura 10 - Cobogó: unión de la tradición con tecnologías industrializadas

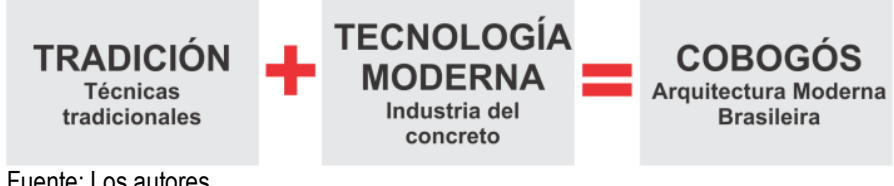

Fuente: Los autores

Esta estrategia, al igual que todos los elementos vazados como lo manifiesta Holanda (1976), permite la ventilación e iluminación natural, así como también filtra la incidencia solar y permite obtener privacidad en el interior de los ambientes (Figura 11); un instrumento de adaptación tropical utilizado antes de la introducción de equipamientos mecanizados, como el aire acondicionado.

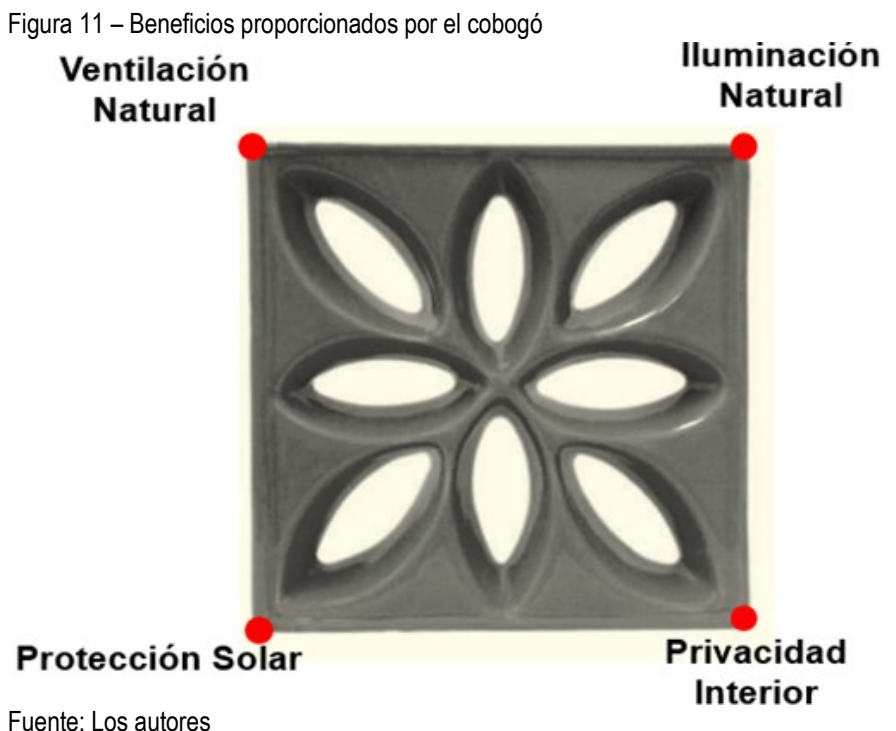

Bruand (2010), manifiesta que, en 1935, Luís Nunes, fue el primer arquitecto en utilizar este elemento arquitectónico (cobogó) en el proyecto (Figura 12) denominado "Caixa d' água" en la ciudad de Olinda. Este acontecimiento marca un precedente en la arquitectura moderna brasilera, no sólo a nivel local, si no también nacional; pues a partir de esta innovación, este elemento fue incorporado en importantes proyectos modernos, como los desarrollados posteriormente por Rino Levi en el Instituto Sedes Sapientiae en 1940 (Figura 13), Affonso Reydy en la del Conjunto Residencial Pedregulho en 1947 (Figura 14), Lúcio Costa en los edificios del Parque Eduardo Guinle en 1948 (Figura 15), Oscar Niemeyer en la obra de la Escuela Estadual Julia Kubitschek en 1951(Figura 16), Marcio Kogan en la Casa Cobogó (figura 17), etc.

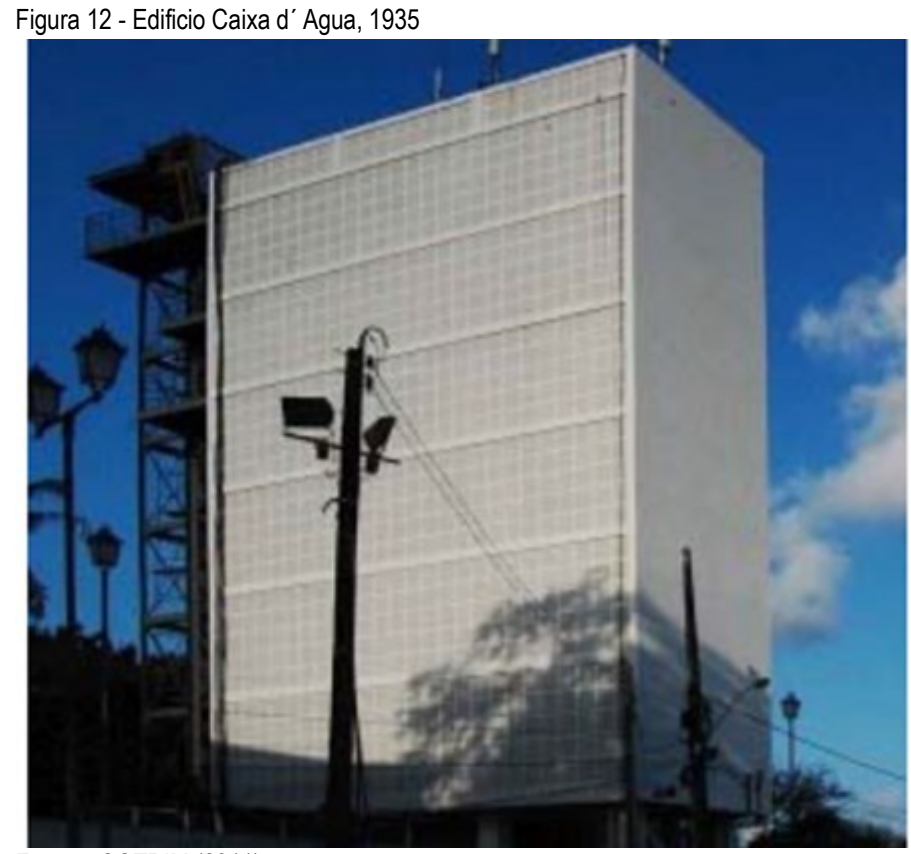

Fuente: COTRIM (2011)

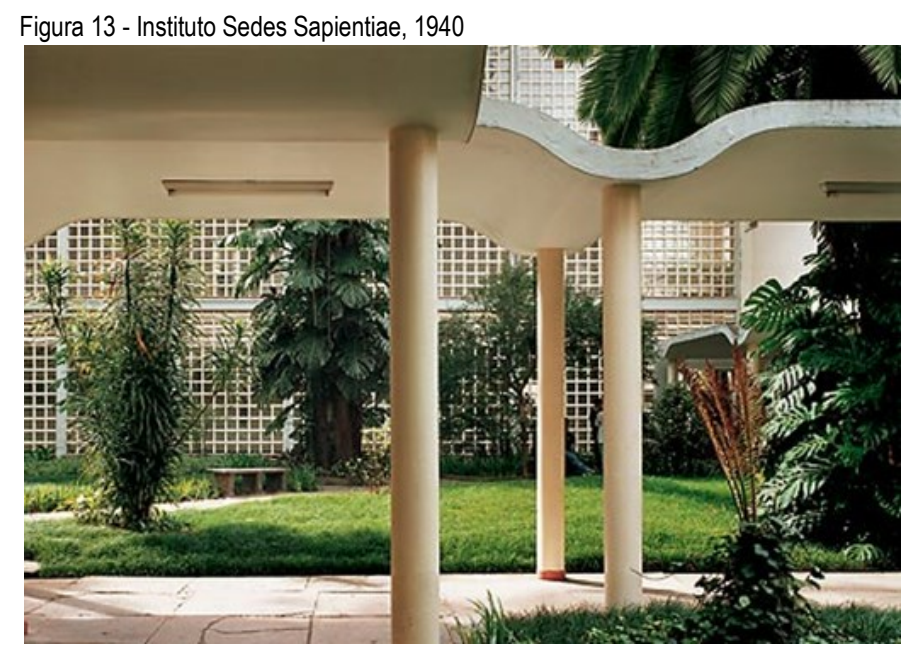

Fuente: KON (S/D) 
Figura 14 - Conjunto Residencial Pedregulho, 1947

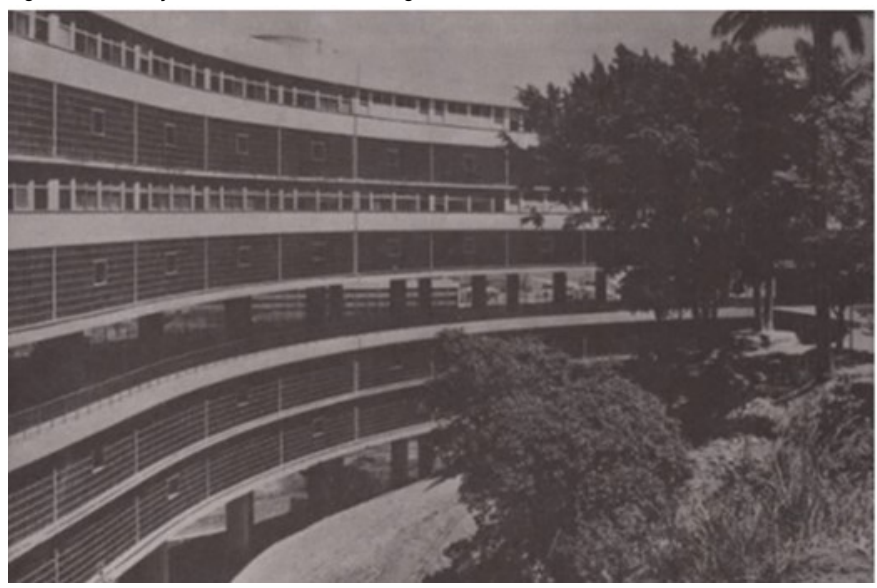

Fuente: BRUAND (2010)

Figura 15 - Edificios del Parque Guinle, 1948

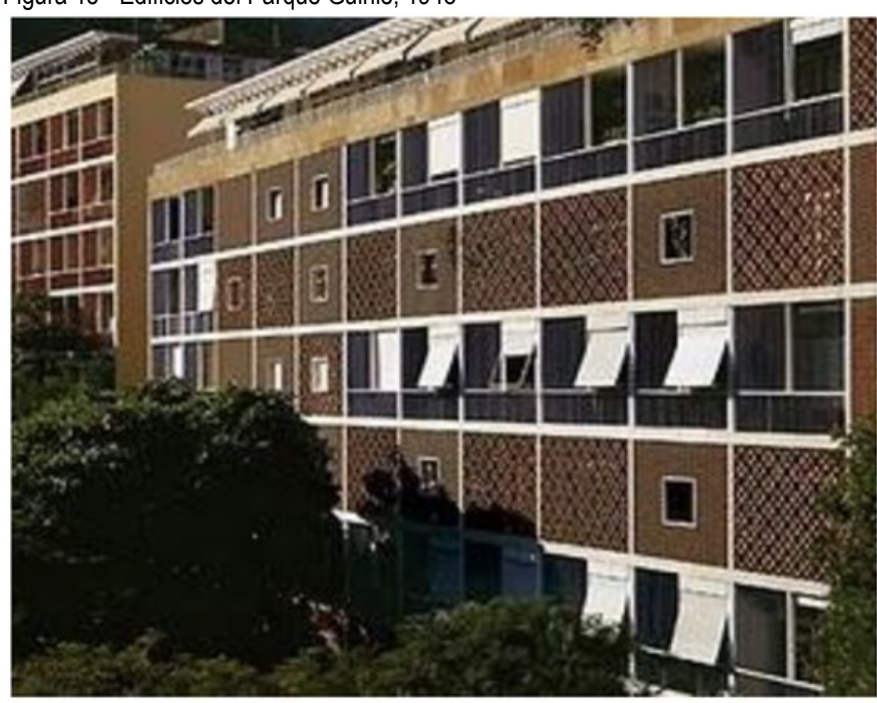

Fuente: WISNIK (2001)

Figura 16 - Escuela Estadual Julia Kubitschek, 1951

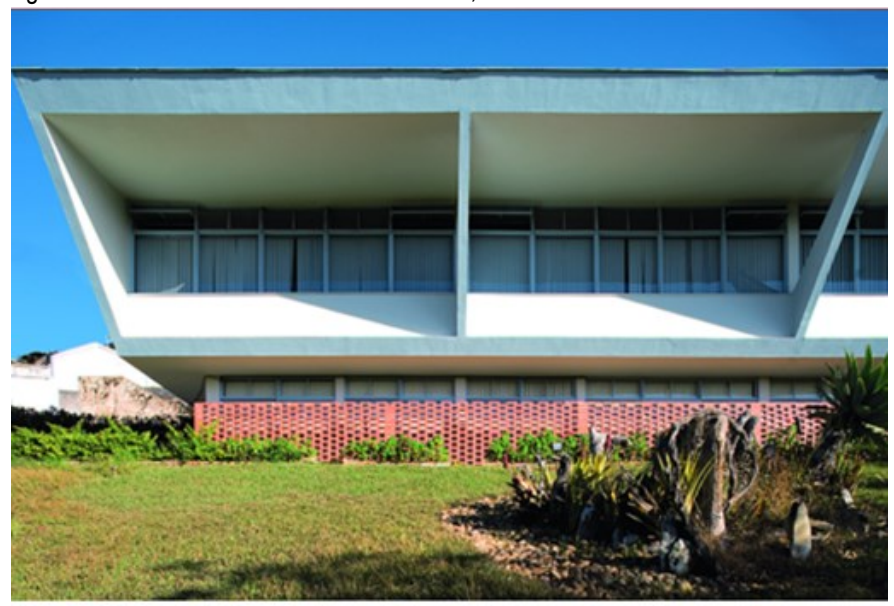

Fuente: FINOTTI (S/D)
Figura 17 - Casa Cobogó, 2011

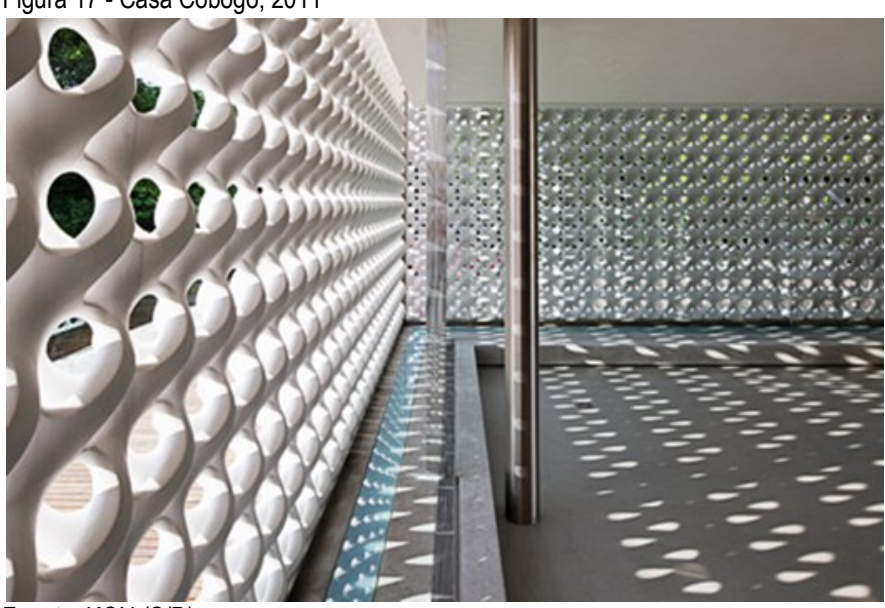

Fuente: KON (S/D)

Originalmente, los cobogós fueron concebidos con un diseño que posibilitó la producción en serie de las piezas, tal elemento consistía en un ladrillo hecho de concreto, midiendo $0,50 \mathrm{~m} \times 0,50 \mathrm{~m} \times 0,10 \mathrm{~m}$ con perforaciones cuadradas de $0,05 \mathrm{~m}$. Actualmente estos elementos son encontrados de diferentes modelos y hechos de diferentes materiales tales como cerámica, losa, porcelana, aluminio, vidrio etc.

Los cobogós, a partir de su primera utilización en 1935, se convirtieron en una constante en los proyectos tanto públicos como privados, siendo usados como cerramientos de grandes fachadas como así lo demuestra la Tabla 1 que presenta un resumen cronológico por décadas, de las obras arquitectónicas brasileras significativas, que utilizaron en su composición arquitectónica el cobogó, demostrando el tipo de material que fue utilizado, el uso de la edificación (público o privado); además del lugar y el arquitecto que proyecto y/o construyó.

Es posible percibir en la tabla 1, entre las edificaciones significativas analizadas, el cobogó tuvo su uso intensificado hasta el final de la década de 1950, siendo nuevamente utilizado con más intensidad después de los años 2000. En cuanto al material utilizado para la fabricación del cobogó, predomina el concreto (52\%), seguido por otros materiales $(31 \%)$ y por el cerámico que es significativamente menor que los demás (17\%). En esta perspectiva, el uso del cobogó cerámico se inició a mediados de la década de 1940 y la adopción de nuevos materiales a mediados de la década de 1950. 
Tabla 1. Histórico de utilización de elementos perforados en Obras relevantes de la Arquitectura Brasilera

\begin{tabular}{|c|c|c|c|c|c|c|c|c|c|c|}
\hline \multirow[b]{2}{*}{ DECADA } & \multirow[b]{2}{*}{ AÑOS } & \multirow{2}{*}{ NOMBRE DEL PROYECTO } & \multicolumn{3}{|c|}{ MATERIAL DEL COBOGÓ } & \multicolumn{2}{|c|}{ USO } & \multirow{2}{*}{$\begin{array}{l}\text { ARQUITETO O } \\
\text { EMPRESA }\end{array}$} & \multicolumn{2}{|c|}{ LOCALIZACIÓN } \\
\hline & & & Concreto & Cerámico & Otros & Público & Privado & & Ciudad - Estado & Región \\
\hline \multirow{7}{*}{1930} & 1935 & Caixa d'Água de Olinda & $x$ & & & $x$ & & Luiz Nunes & Pernambuco - PE & Nordeste \\
\hline & 1938 & da Mirueira & $x$ & & & $x$ & & Luiz Nunes & Pernambuco - PE & Nordeste \\
\hline & 1938 & Grande Hotel de Ouro Preto & $x$ & & & & $\mathrm{X}$ & Oscar Niemeyer & Ouro Preto - MG & Sudeste \\
\hline & 1940 & Instituto Sedes Sapientiae & $\mathrm{X}$ & & & $X$ & & Rino Levi & Sao Paulo - SP & Sudeste \\
\hline & 1944 & Colônia de Férias da Tijuca & $x$ & & & $x$ & & MMM Roberto & Rio de Janeiro - RJ & Sudeste \\
\hline & & Residencial Mendes de & & & & & & Affonso Eduardo & & \\
\hline & 1947 & Moraes (Pedregulho) & & $x$ & & & $x$ & Reidy & Rio de Janeiro - RJ & Sudeste \\
\hline 1940 & 1948 & $\begin{array}{l}\text { Edifícios Parque Eduardo } \\
\text { Guinle }\end{array}$ & & $x$ & & & $x$ & Lúcio Costa & Rio de Janeiro - RJ & Sudeste \\
\hline \multirow{11}{*}{1950} & & & & & & & & Olavo Redig de & & \\
\hline & 1950 & Instituto Moreira Salles & $x$ & & & $x$ & & Campos & Rio de Janeiro - RJ & Sudeste \\
\hline & 1951 & Residência no Morumbi & $x$ & & & & $x$ & Oswaldo Bratke & Sao Paulo - SP & Sudeste \\
\hline & & Escola Estadual Professora & & & & & & & & \\
\hline & 1951 & Júlia Kubitschek & $x$ & & & $x$ & & Oscar Niemeyer & Minas Gerais - MG & Sudeste \\
\hline & 1951 & Hotel Tijuco - Diamantina & $x$ & & & & $x$ & Oscar Niemeyer & Minas Gerais - MG & Sudeste \\
\hline & 1953 & Edifício União & & $x$ & & & $x$ & Acacio Gil Borsoi & Recife - PE & Nordeste \\
\hline & 1954 & Edifício Eiffel & $x$ & & & & $x$ & Oscar Niemeyer & Sao Paulo - SP & Sudeste \\
\hline & 1953 & Instituto de Antibióticos & & & $x$ & $x$ & & Mário Russo & Pernambuco - PE & Nordeste \\
\hline & 1958 & $\begin{array}{l}\text { Residencia de Isnard Castro } \\
\text { e Silva }\end{array}$ & & & $x$ & $x$ & & Joaquim Guedes & Sao Paulo - SP & Sudeste \\
\hline & 1959 & $\begin{array}{l}\text { Residencia Castor Delgado } \\
\text { Perez }\end{array}$ & & $x$ & & & $x$ & Rino Levi & Sao Paulo - SP & Sudeste \\
\hline \multirow{3}{*}{1960} & & Edifício do Seminário Reg. & & & & & & Fernandes & & \\
\hline & 1962 & do Nordeste & & & $x$ & $x$ & & Amorim & Pernambuco - PE & Nordeste \\
\hline & 1963 & Edifício Santo Antonio & & & $x$ & & $\mathrm{X}$ & Acácio Gil Borsoi & Recife - PE & Nordeste \\
\hline 1970 & 1970 & - & - & - & - & - & - & - & - & - \\
\hline 1980 & 1981 & Casa Guarujá & & & $x$ & & $x$ & $\begin{array}{l}\text { Paulo Mendes da } \\
\text { Rocha }\end{array}$ & Sao Paulo - SP & Sudeste \\
\hline 1990 & 1990 & $\begin{array}{l}\text { edifício esportivo SESC- } \\
\text { Pompéia }\end{array}$ & & $x$ & & $\mathrm{X}$ & & Lina Bo Bardi & Sao Paulo - SP & Sudeste \\
\hline \multirow{9}{*}{2000} & 2003 & Casa Pinheiros & $\mathrm{X}$ & & & & $\mathrm{x}$ & Isay Weinfeld & Sao Paulo - SP & Sudeste \\
\hline & & Escola de Ensino & & & & & & MMBB Arquitetos & & \\
\hline & 2003 & Fundamental FDE Campinas & $\mathrm{x}$ & & & $x$ & & IVIIVIBD Arquitetos & Sao Paulo - SP & Sudeste \\
\hline & 2006 & Casa Iporanga & & & $x$ & & $x$ & Isay Weinfeld & Sao Paulo - SP & Sudeste \\
\hline & & Colégio Prof. Marcos & & & & & & EGME Arruitetoc & & \\
\hline & 2008 & Alexandre Sodré & $x$ & & & $x$ & & FGIVIF Arquitetos & Sao Paulo - SP & Sudeste \\
\hline & & Instituto de Química da & & & & & & Aleixo Furtado e & & Cen- Oeste \\
\hline & 2008 & Universidade de Brasília & $\mathrm{X}$ & & & $x$ & & Marcílio Ferreira. & Brasilia- DF & \\
\hline & 2008 & $\begin{array}{l}\text { Biblioteca Nacional de } \\
\text { Brasília }\end{array}$ & $x$ & & & $x$ & & Lúcio Costa & Brasilia- DF & Cen- Oeste \\
\hline \multirow{3}{*}{2010} & 2011 & Casa Cobogó & & & $x$ & & $x$ & Márcio Kogan & Sao Paulo - SP & Sudeste \\
\hline & 2013 & Casa Jardins & & & $x$ & & $x$ & CR2 Arquitetura & Sao Paulo - SP & Sudeste \\
\hline & 2017 & Edificio Japan House & & & $\mathrm{X}$ & $\mathrm{X}$ & & Kengo Kuma & Sao Paulo - SP & Sudeste \\
\hline TOTAL & - & - & 15 & 5 & 9 & 15 & 14 & - & - & - \\
\hline
\end{tabular}

${ }^{*}$ OBS. Considerase otros materiales al vidrio, madera, losa, porcelana, etc.

Fuente: Los autores.

\section{Tendencias y Perspectivas para el uso del Cobogó}

Desde su creación (elaboración artesanal), hasta la actualidad (reproducción industrial); el cobogó ha pasado por diferentes modificaciones, desde la elaboración de diseños simples con materiales como concreto $\mathrm{y} / \mathrm{o}$ cerámica, hasta la elaboración computacional de sofisticados y complejos elementos producidos con aluminio $\mathrm{u}$ otro metal, que pueden ser doblados $\mathrm{y}$ adaptados fácilmente.
Actualmente el mercado brasileiro, ofrece cobogós de distintos materiales, con perforaciones de diseños y dimensiones variadas; este elemento se ha convertido en un componente industrial costoso, a pesar de haber sido concebido como un elemento de fácil uso, elaboración y adquisición. Hoy en día, en muchos casos, se convierte en un elemento de altísima complejidad geométrica, incorporado en proyectos muchas veces, no por su eficiencia en cuestiones de aprovechamiento energético, si apenas por cuestiones estéticas, o sea no por cuestiones funcionales y si por cuestiones formales. 
Las investigaciones apuntan que actualmente (2017), existe una tendencia de uso en ambientes internos, este uso muchas veces solo se limita a actuar como separadores de ambiente con predominancia en el aspecto decorativo y no funcional, así mismo, el uso interno, desde que bien planificado, auxilia en la ventilación entre ambientes, en la privacidad y en la distribución de la luz.

Hensel (2008), defiende la actualización contemporánea, de este tipo de estrategias arquitectónicas de control ambiental, pues estas son estrategias que dialogan con el medio ambiente, además de proporcionar soluciones deseadas con respecto al aprovechamiento de la ventilación e iluminación natural, trayendo consigo un mejor desempeño energético.

Se visualiza que este elemento, es una estrategia arquitectónica que puede ser incorporado en la concepción de proyectos con el objetivo de buscar una conciliación entre ambiente construido y clima, para tal efecto se debe recurrir a sus orígenes y rescatar la función y el objetivo por el cual fue creado estableciendo una integración entre forma y función.

\section{Consideraciones Finales}

Mediante el análisis realizado de la bibliografía escogida, con la utilización de los filtros anteriormente citados en la metodología, se comprueba que el cobogó es de mucha importancia para la arquitectura, por ser un elemento que aporta ventilación e iluminación natural, así como protección solar y privacidad en el ambiente interno. Un elemento arquitectónico que nace en la arquitectura brasilera gracias a la influencia de muxarabis, rotulas y celosías.

Este tipo de estrategia fue desarrollada ya que, desde la antigüedad, siempre hubo una preocupación por la adaptación a las condiciones climáticas locales, principalmente con relación al control de la radicación solar directa. De este modo la arquitectura brasilera, utilizó con gran sabiduría este tipo de elementos desde el periodo de la colonización (Siglo XVI), hasta los días actuales (siglo XXI).

A pesar de todos los cambios culturales en este periodo, de los modos de vida, de patrones, tecnologías y técnicas constructivas, los elementos perforados nunca fueron olvidados, por el contrario, se fueron adaptando $\mathrm{y}$ modernizando, adquiriendo nuevos formatos y fabricados de nuevos materiales.

En este contexto, uno de los grandes avances fue el desarrollo del cobogó a finales de la década de 1920. A través del uso de materiales modernos como el cemento, integrado a las necesidades actuales de racionalidad constructiva, modulación y fabricación en serie.

Con el pasar del tiempo, este elemento también se fue modificando, de una geometría simple, pasó a ser elaborado con geometrías más complejas, del cemento pasó a la cerámica cocida y posteriormente realizado con mayores acabados y por consecuencia convirtiéndose en un elemento muchas veces costoso. Su forma de utilización también sufrió alteración a lo largo de su existencia, pasando de su uso únicamente en fachadas externas de la edificación a ser inserido o integrar también los espacios internos de los ambientes de una edificación.

Por otra parte, es importante dar a conocer que actualmente existe una reducida bibliografía actualizada sobre datos técnicos precisos que nos den a conocer la relación que existe entre la geometría y la función del elemento, las investigaciones muchas veces se resumen a dar a conocer el uso de los elementos perforados en proyectos de arquitectura de una manera general, resaltando la forma estética que el elemento en cuanto a su diseño presenta.

En los estudios realizados, no se analiza si el diseño propuesto o escogido dentro de los existentes en el mercado, proporciona un adecuado desempeño con relación a la ventilación e iluminación natural, tomando como referencia normas técnicas, que presenten parámetros de ventilación e iluminación, en los ambientes internos de una edificación. Este tipo de dificultades ocurre muchas veces, porque no se le toma la debida importancia a este elemento de origen brasileño, que surgió en la arquitectura moderna, gracias a una adecuada reinterpretación de las técnicas constructivas tradicionales y la aplicación de tecnología moderna de la industria del concreto.

\section{Agradecimientos}

Los autores agradecen a la Coordinación de Aperfeccionamiento de Personal de Nivel Superior (CAPES), por el soporte financiero para el desenvolvimiento de la presente investigación.

\section{Referencias}

AFONSO, A. Revolução na arquitetura: Recife, década de Trinta. Teresina: EDUFPI, 2001. 
ARAÚJO, C. V. de A. Análise de componentes arquitetônicos para potencialização da ventilação natural com ênfase em captadores de vento. Dissertação (Mestrado em Conforto no Ambiente Construído; Forma Urbana e Habitação) - Universidade Federal do Rio Grande do Norte, Natal, 2011.

BITTENCOURT, L. Efeito da Forma dos Elementos Vazados na Resistência Oferecida à Passagem da Ventilação Natural. In: ENCONTRO NACIONAL DE CONFORTO NO AMBIENTE CONSTRUÍDO, 3 Gramado, 1995. Anais...

Disponible en: http://www.infohab.org.br/acervos/buscaautor/codigoAutor/3979

Acceso en: 30 Ene. 2018.

BRAGA, M. O concurso de Brasília: sete projetos para uma capital. São Paulo, 2010.

BRASIL, Ministério de Minas e Energia - MME. Empresa de Pesquisa Energética - EPE. Balanço Energético Nacional 2017 (ano base 2016) - Relatório Final 2017. Disponible en: https://ben.epe.gov.br/downloads/Relatorio_Final_BEN_2017.pdf Acceso en: 30 Ene. 2018.

BRUAND, Y. Arquitetura contemporânea no Brasil. Ed. Perspectiva. São Paulo. 2010.

COLIN, S. Técnicas construtivas do período colonial. Coisas da Arquitetura.

Disponible en: https:/coisasdaarquitetura.wordpress.com/2010/09/06/tecnicas-construtivas-do-periodo-colonial-ii/ Acceso en: 30 Ene. 2018.

COTRIM, M. Fotografia do Reservatório d'Agua de Olinda. In: Eu vi o modernismo nascer... foi no Recife. Revista Vitruvius, ${ }^{\circ}$ 131. 02. 2011.

CHING, D. K. Dicionário visual de arquitetura. São Paulo: Martins Fontes, 2000.

MELLO, E. K. de. A herança mourisca da arquitetura no Brasil. Universidade de São Paulo, Faculdade de Arquitetura e Urbanismo, 1974

ELLA NETWORK. Adaptación y mitigación del cambio climático en zonas urbanas. 2013. Disponible en: http:/ella.practicalaction.org/es/knowledge-guide/city-level-climate-change-mitigation-and-adaptation/. Acceso en: 30 Ene. 2017.

EL-SHORBAGY, A. M. Traditional Islamic-Arab house: vocabulary and syntax. International Journal of Civil \& Environmental Engineering IJCEE-IJENS, 2010.

EPE- Empresa de Pesquisa Energética. Balanço Energético Nacional 2017 (año base 2016) - Relatório Final 2017. Disponible en: https://ben.epe.gov.br/downloads/Relatorio_Final_BEN_2017.pdf. Acceso en: 30 Ene. 2018.

FATHY, H. Architecture for the poor: an experiment in rural Egypt. University of Chicago, 2010.

FERREIRA, A. B. H. Novo dicionário Aurélio da língua portuguesa. 3. ed. São Paulo: Positivo, 2004.

FICARELLI, L. The Domestic Architecture in Egypt between Past and Present: The Passive Cooling in Traditional Construction. En THIRD INTERNATIONAL CONGRESS ON CONSTRUCTION HISTORY. Proceedings... Berlin Neunplus1, 2009.

FINOTTI, L. Escola Est. Professora Júlia Kubitschek in: fotografia, Revista Projeto Design na Edição. 334. São Paulo. Acceso en: 30 Ene. 2018.

GALVÃO, M. A. Pilar de Goiás, do século XVIII ao XXI. Arquiteturismo, São Paulo, año 02, n. 020.05, Vitruvius, out. 2008. Disponible en: http://www.vitruvius.com.br/revistas/read/arquiteturismo/02.020/1468 Acceso en: 30 Ene. 2018.

GOMEZ, L. S. R. Os 4P's do Design: Uma proposta metodológica não linear de projeto. Tese (Doutorado) -PPGEP-UFSC, Florianópolis, 2004.

GOMES, R. V. O Magrebe e o Gharb al-Andaluz: testemunhos arqueológicos e simetrias culturais (séculos VIII-XIII). Camões Revista de Letras e Culturas Lusófonas. v. 17-18, p. 110-124. 2004. ISSN 0874-3029

GOODWIN, P. L. Brazil builds: architecture old and new: 1652-1942. Nova York: The Museum of Modern Art, 1943. 
GUERRA, M. R. Arquitectura Bioclimática como parte fundamental para el ahorro de energía en edificaciones. Ed., Don Bosco, El Salvador, 2013.

HENSEL, M. Performance-Oriented Design: Precursors and Potentials. Londres: Architectural Design, 2008.

HOLANDA, A de. Roteiro para construir no Nordeste. Arquitetura como lugar ameno nos trópicos ensolarados. Recife: UFPE, 1976.

IEA - INTERNATINAL AGENCY ENERGY. Atlas of Energy In: Atlas: Energy Balance. Disponible en: http://energyatlas.iea.org/\#!/tellmap/-1002896040

Acceso en: 30 Ene. 2018.

KON, N. Imagens de Arquitetura e Cidades In: Arquiteturas Brasileira Moderna e Contemporânea. São Paulo S/D.

Disponible en: http://www.nelsonkon.com.br/entrada.asp Acceso en: 30 Ene. 2018.

LEMOS, C. A. C. Uma nova proposta de abordagem da história da arquitetura brasileira. Arquitextos, São Paulo, año 12, n. 141.00, Vitruvius, fev. 2012 Disponible en: http://www.vitruvius.com.br/revistas/read/arquitextos/12.141/4214. Acceso en: 30 Ene. 2018.

MARCONI, M. D. A.; LAKATOS, E. M. Fundamentos de metodología científica. 5. ed.-São Paulo: Atlas. 2003.

MARIANNO, J. Influencias muçulmanas na architectura tradicional brasileira. Editora A Noite, 1943.

MINDLIN, H. Arquitetura moderna no Brasil. Ed. Colibris. Rio de Janeiro, 1956.

NERY, E. A obra em tempos vários. Recife, 1999.

PALLONE, S. A arquitetura da felicidade: beleza ou funcionalidade? Um passeio pela história e pela filosofia da arquitetura oferece algumas pistas. 2010.

PAULERT, R. Uso de elementos vazados na arquitetura: Estudo de três obras educacionais contemporâneas. Dissertação de mestrado Universidade Federal do Paraná. Curitiba, 2012.

PINTO, E.; FREYRE, G. Muxarabis \& balcões: e outros ensaios. Companhia Editora Nacional. 1958.

RABBAT, R. M. C. Introdução ao desenvolvimento de esquadrias de madeira de eucalipto para habitação de interesse social. São Paulo. 1988.

RODRIGUES, J. W. Documentário arquitetônico: relativo à antiga construção civil no Brasil. Ed. Itatiaia, 1979 .

SILVA, G. G. Marcos da arquitetura moderna em Pernambuco. In: Revista Projeto. Edição especial. 1988.

WISNIK, G. Lúcio Costa. Ed. Cosac Naify. São Paulo. 2001.

\footnotetext{
${ }^{1}$ Darwin Onésimo Jaime Camacho

Arquitecto y Urbanista. Dirección postal: Beco Mário Lamarque nº 06, apartamento 06, Centro, Foz de Iguazú, PR, Brasil, 85851017
}

\section{${ }^{2}$ Helenice María. Sacht}

Arquitecta y Urbanista. Doctora en Ingeniería Civil. Dirección postal: Avenida Tancredo Neves 6731, Bloque 06, Espacio 01, Sala 10, Conjunto B, Foz de Iguazú, PR, Brasil, 85867-970.

\section{${ }^{3}$ Egon Vettorazzi}

Arquitecto y Urbanista. Maestro en Ingeniería Civil y Ambiental. Dirección postal: Avenida Tancredo Neves 6731, Bloque 06, Espacio 01, Sala 2, Conjunto B, Foz de Iguazú, PR, Brasil, 85867-970. 